\title{
Tratamento cirúrgico da comunicação interventricular pós infarto agudo do miocárdio: conduta atual
}

\author{
Luís Alberto DALLAN*, Sérgió de Almeida OLIVEIRA*, José Antônio F. RAMIRES*, Alexandre SABINO \\ NETO*, Geraldo VERGINELLI*, Adib D. JATENE*
}

DALLAN, L. A.; OLIVEIRA, S. A.; RAMIRES, J. A. F.; SABINO NETO, A.; VERGINELLI, G.; JATENE, A. D. - Tratamento cirúrgico da comunicação interventricular pós infarto agudo do miocárdio: conduta atual. Rev. Bras. Cir. Cardiovasc., 4(1): 64-74, 1989.

RESUMO: Os autores descrevem sua experiência com o tratamento cirúrgico de 42 pacientes portadores de comunicação interventricular (CIV) pós infarto agudo do miocárdio (IAM). Destacam a elevada mortalidade cirúrgica nos pacientes com choque cardiogênico instalado $(66,6 \%)$ em relação aos demais $(9,5 \%)$. A observaçāo de descompensaçōes súbitas em pacientes hemodinâmicamente estáveis tem levado à indicação cirúrgica precoce, se possível, assim que estabelecido o diagnóstico da rotura do septo interventricular (RSI). O comprometimento de múltiplas artérias coronárias e faixas etárias elevadas foram considerados fatores agravantes no prognóstico cirúrgico. O mesmo não ocorreu com a relação fluxo pulmonar/sistêmico e shunt E-D, que não guardaram relação com a mortalidade. A técnica de exposição de ambas as cavidades ventriculares e reforço das 2 faces do septo roto com tecido biológico tem fornecido resultados gratificantes na correçăo da RSI, especialmente de localização posterior. cirurgia.

DESCRITORES: rotura ventricular, cirurgia; comunicação interventricular, cirurgia; infarto do miocárdio,

\section{INTRODUÇÃO}

A rotura do septo interventricular (RSI) é uma complicaçāo grave do infarto agudo do miocárdio (IAM)

Sua incidência, observada por diversos autores, varia de $0,5 \%$ a $3 \%{ }^{15}, 25 .{ }^{33}$, sendo responsável pelo óbito de $1 \%$ a $5 \%$ do total dos pacientes infartados ${ }^{7}$.

A evolução natural da doença demonstra a ineficácia do tratamento clínico isolado ${ }^{32,}{ }^{33}$. Calcula-se mortalidade de $24 \%$ nas primeiras 24 horas de sua instalação, $46 \%$ na primeira semana, $65 \%$ em 2 semanas, $67 \%$ a $87 \%$ em 2 meses e sobrevida de apenas 5 a $7 \%$ ao final de 1 ano $7,10,35,38$.
Essas evidências elegeram a correção cirúrgica como tratamento obrigatório. Persistem, entretanto, controvérsias quanto ao momento ideal para tal intervenção. Chegou-se a recomendar a espera mínima de 3 semanas após o IAM, período suficiente para o desenvolvimento de fibrose no tecido miocárdico, visando facilitar a correção cirúrgica e prevenir eventuais recidivas ${ }^{8,20}$. A contrapulsação com balăo intra-aórtico, o uso de drogas vasodilatadoras e de drogas inotrópicas contribuem para a estabilizaçăo hemodinâmica - ainda que transitória de alguns desses pacientes, postergando a intervenção cirúrgica. Entretanto, logo se observou que a açăo das drogas vasodilatadoras visando reduzir a pós-carga ventricular também proporciona vasodilatação pulmonar, al-

Trabalho realizado no Instituto do Coração do Hospital das Clínicas da Faculdade de Medicina da Universidade de São Paulo. Săo Paulo, SP. Brasil.

Apresentado ao 16: Congresso Nacional de Cirurgia Cardiaca. São Paulo, SP, 7 e 8 de abril, 1989

* Do Instituto do Coraçáo do Hospital das Clínicas da Faculdade de Medicina da Universidade de Săo Paulo.

Endereço para separatas: Luis Alberto Dallan. Av. Dr. Enéas de Carvalho Aguiar, 44. Divisăo Cirúrgica. 05403 São Paulo, SP, Brasil. 
DALLAN, L. A.; OLIVEIRA, S. A.; RAMIRES, J. A. F.; SABINO NETO, A.; VERGINELLI, G.; JATENE, A. D. - Tratamento cirúrgico da comunicação interventricular pós infarto agudo do miocárdio: conduta atual. Rev. Bras. Cir. Cardiovasc., 4(1): 64-74, 1989.

gumas vezes superior à sistêmica, aumentando o fluxo E-D ${ }^{38}$. Além disso, sua administraçāo geralmente acarreta ao paciente hipotensão severa, comprometendo ainda mais seu estado geral. Os pacientes mais graves freqüentemente necessitam quantidades maiores de drogas vasopressoras e inotrópicas, o que predispōe a taquiarritmias, maior isquemia miocárdica e vasoconstrição periférica, com elevação do fluxo E-D ${ }^{33}$.

Essas observaçōes evidenciaram a fragilidade dessa aparente estabilizaçāo hemodinâmica, demonstrando sua utilidade para eventual preparo pré-operatório do paciente, mas desestimulando o tratamento clínico por longo período.

A ocorrência de descompensação súbita em pacientes "hemodinamicamente estáveis", na primeira, ou mesmo na segunda semana pós RSI, levado a intervenção de urgência em condiçōes desfavoráveis, também contribuiu para firmar o conceito de indicação cirúrgica mais precoce ${ }^{27,29}$.

A elevada mortalidade clínica e cirúrgica traduz bem a gravidade dessa complicação do IAM, descrita por diversos autores como catastrófica ${ }^{23}, 30,38$. REDFORD et alii ${ }^{33}$ observaram mortalidade cirúrgica hospitalar global de $39 \%$, que se reduzia para $18 \%$ em pacientes operados sem choque cardiogênico instalado. NAIFEH et alii ${ }^{30}$ obtiveram $54 \%$ de mortalidade, especialmente naqueles pacientes cuja RSI localizou-se na região posterior do septo. Em nosso meio, NESRALLA et alii ${ }^{31}$ observaram mortaliade de $33 \%$ dos 9 pacientes submetidos a correção cirúrgica de RSI. PIEGAS et alii ${ }^{32}$ obtiveram $28 \%$ de mortalidade, dentre 18 pacientes operados em igual situação.

Nossa experiência com 42 pacientes operados nos últimos 5 anos e meio nos permite identificar fatores associados à maior morbidade e mortalidade, assim como evidenciar melhores resultados com a abordagem biventricular no fechamento da RSI, especialmente em roturas localizadas na porção posterior do septo.

\section{CASUÍSTICA E MÉTODOS}

No período de maio de 1983 a novembro de 1988 , 46 pacientes com diagnóstico de RSI pós infarto transmural do miocárdio foram atendidos no Instituto do Coração. Destes, 42 foram submetidos a correção cirúrgica. Os 4 restantes faleceram antes que a operação pudesse ser realizada.

Três pacientes necessitaram reoperação, 2 devido a persistência de comunicação interventricular (CIV) e 1 pelo desenvolvimento de pseudo-aneurisma na ventriculografia esquerda (E).

Os pacientes procederam de diferentes localidades e Serviços, o que propiciou heterogeneidade no período entre a RSI e a intervenção cirúrgica.
Constatamos que a CIV incidiu mais no sexo masculino $(61,9 \%)$. $A$ idade variou entre 47 e 79 anos $(M=$ 65,38 anos).

Trinta e nove (92,9\%) pacientes eram de cor branca, $2(4,8 \%)$ de cor negra e $1(2,4 \%)$ de cor amarela.

O diagnóstico da RSI foi feito pela presença de sopro sistólico precordial e corroborado em todos os pacientes por exame cinecoronariográfico (Figura 1). Estudos complementares rotineiros como radiografia de tórax, eletrocardiograma, ecocardiograma, medidas pressóricas com cateteres de Swan-Ganz e determinaçōes enzimáticas também foram realizados.

Em $28(66,7 \%)$ pacientes a RSI ocorreu na primeira semana do IAM, sendo $5(11,9 \%)$ no mesmo dia; em $6(14,3 \%)$ pacientes a RSI ocorreu na segunda semana; em $3(7 \%)$ na segunda quinzena e em $5(11,9 \%)$ não se pôde determinar seu aparecimento.

Vinte $(47,6 \%)$ pacientes apresentavam apenas comprometimento uniarterial (lesão obstrutiva com estreitamento igual, ou superior, a $70 \%)$, sendo $14(70 \%)$ de artéria descendente anterior (DA) e $6(30 \%)$ de coronária direita (CD). Em $19(23,8 \%)$, havia comprometimento biarterial e, nos demais $12(28,6 \%)$, triarterial.
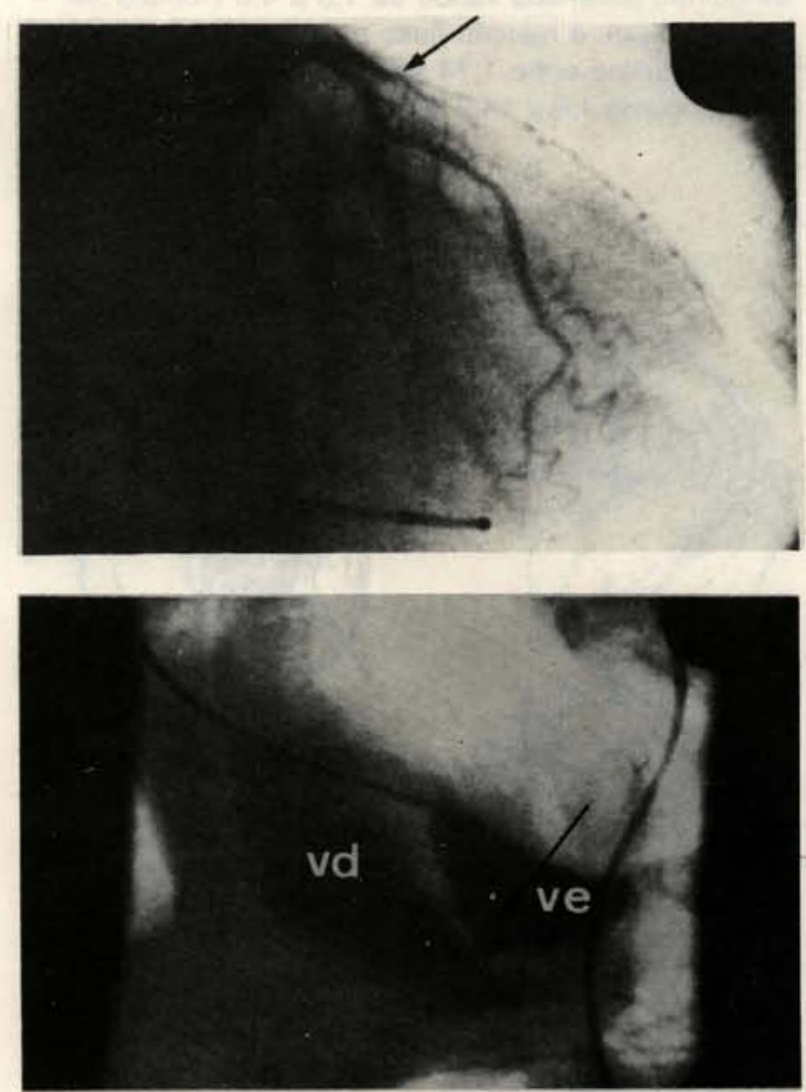

Fig. 1 - Superior: coronariografia E demonstrando a oclusāo da artéria descendente anterior, que ocasionou a RSI. Inferior: passagem de contraste VE-VD através de CIV anterior. 
DALLAN, L. A.; OLIVEIRA, S. A.; RAMIRES, J. A. F.; SABINO NETO, A.; VERGINELLI, G.; JATENE, A. D. - Tratamento cirúrgico da comunicação interventricular pós infarto agudo do miocárdio: conduta atual. Rev. Bras. Cir. Cardiovasc., 4(1): 64-74, 1989.

A correlação entre ventriculografia e ECG demonstrou que a rotura ocorreu na porçāo anterior ou apical do septo nos $28(66,6 \%)$ pacientes com infarto anterior, e na porçăo posterior do septo nos $14(33,3 \%)$ com infarto inferior.

Vinte e quatro $(57,1 \%)$ pacientes eram portadores de aneurisma do ventrículo esquerdo, $3(12,5 \%)$ localizados em parede inferior.

Em 30 pacientes, o acesso à CIV foi feito através de ventriculotomia E. Nesses pacientes, empregou-se retalho de Teflon ou pericárdio bovino suturado diretamente no septo interventricular $E$ de maneira a fechar a CIV. Destes, $22(73,3 \%)$ eram portadores de RSI anterior e $8(26,6 \%)$ de RSI posterior. Nos outros 12, as cavidades ventriculares $D$ e $E$ foram expostas através da ampliação da CIV, ou biventriculotomia. Isso permitiu a sutura de placas de pericárdio bovino nas faces $D$ e E do septo IV, reforçando-o (Figura 2). O fechamento ventricular foi realizado com o apoio de 2 barras de Teflon envolvendo os retalhos de pericárdio bovino, proporcionando maior reforço à sutura (Figura 3 ).

Em 17 pacientes, o cateterismo pré-operatório possibilitou verificar que o fluxo sangüíneo pulmonar variou de 3,3 a $18,9 \mathrm{l} /$ minuto $(M=7,51 \mathrm{l} /$ minuto); o fluxo sangüíneo sistêmico variou de 1,5 a $4,6 \mathrm{l} /$ minuto $(M)=$ $2,42 \mathrm{l} /$ minuto); a relação fluxo pulmonar/fluxo sistêmico $(\varnothing \mathrm{P} / \mathrm{S})$ oscilou entre 1,74 a $5,87(\mathrm{M}=3,06)$ e o shunt $E-D$ variou de 1,6 a $14,2 \mathrm{l} /$ minuto $(M=5,32 \mathrm{l} /$ minuto).

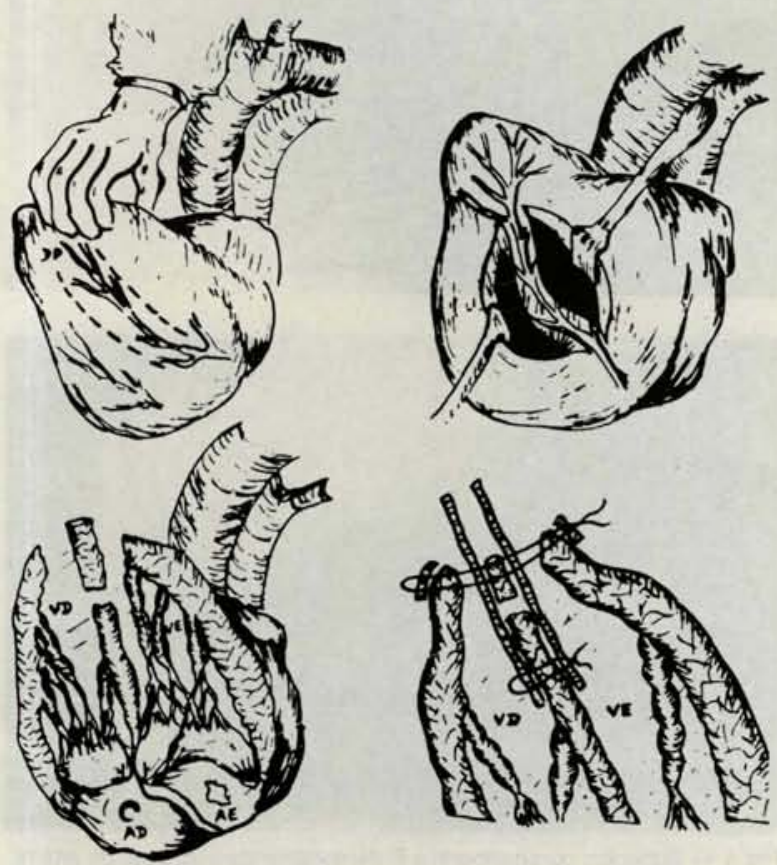

Fig. 2 - Correção de CIV de localização anterior através de biventriculotomia e reforço das 2 faces do septo interventricular.

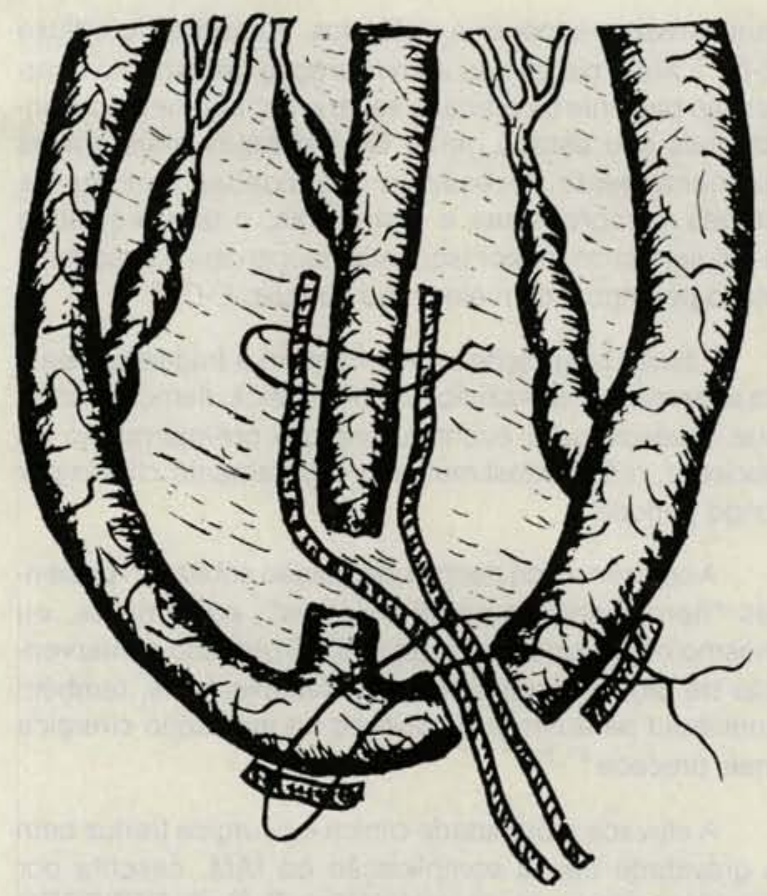

Fig. 3 - Esquema de fechamento de CIV pós IM através de sua ampliaçâo e reforço bilateral do septo com tecido sintético ou biológico.

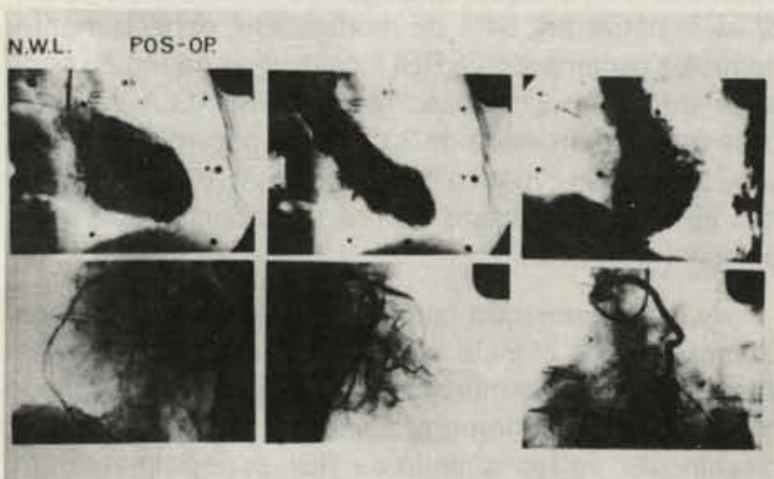

Fig. 4 - Pós-operatório tardio de correção de CIV pós IM. Observa-se a contratilidade preservada de VE e a integridade do septo IV. Ponte de veia safena pérvia para ramo ventricular posterior da artéria circunflexa.

A determinaçāo da saturação de oxigênio em amostras sangüíneas colhidas, respectivamente, de átrio direito (AD), tronco pulmonar (TP) e ventrículo esquerdo (VE) dos pacientes demonstrou salto oximétrico, com valores médios respectivos de: $A D=61,50 \%$, TP $=82,33 \%$, e $V E=94,04 \%$. Esses pacientes foram classificados, quanto à sintomatologia pré-operatória, em grupos funcionais I A IV (critério da New York Heart Association - NYHA), o que possibilitou sua confrontação com os valores de fluxo pulmonar e sistêmico, shunt E-D e mortalidade.

A condição remodinâmica pré-operatória de todos os pacientes foi analisada. Consideramos em choque 
DALLAN, L. A.; OLIVEIRA, S. A.; RAMIRES, J. A. F.; SABINO NETO, A.; VERGINELLI, G.; JATENE, A. D. - Tratamento cirúrgico da comunicação interventricular pós infarto agudo do miocárdio: conduta atual. Rev. Bras. Cir. Cardiovasc., 4(1): 64-74, 1989.

cardiogênico aqueles com hipotensão arterial sistêmica $(\mathrm{M} \leq 60 \mathrm{mmHg})$, recebendo drogas vasopressoras, com má perfusão periférica e débito urinário $\leq 20 \mathrm{ml} / \mathrm{h}$. Vinte e um pacientes foram operados em boas condições hemodinâmicas. Os demais encontravam-se em períodos variáveis de choque cardiogênico. Estes receberam, sistematicamente, balāo intra-aórtico antes ou ao final da operação, sempre que possivel, sua introdução foi localizada através de punção da artéria femoral.

\section{RESULTADOS}

Dos 42 pacientes submetidos a correção cirúrgica da RSI, $16(38,9 \%)$ faleceram no período intra-hospitalar. Dos 26 sobreviventes, 24 (92,3\%) encontram-se assintomáticos, $1(3,8 \%)$ com seqüela neurológica e $1(3,8 \%)$ sem seguimento. Dentre os sobreviventes, 3 necessitaram reoperação, já em fase crônica, sendo 2 por recidiva da CIV e 1 pelo desenvolvimento de pseudo-aneurisma de VE. Os 3 encontram-se assintomáticos.

Não observamos variação estatisticamente significativa de mortalidade, em relação ao sexo. Entretanto, pudemos associar maior mortalidade a faixas etárias mais elevadas; dos 30 pacientes operados com idade inferior a 70 anos, $9(30 \%)$ faleceram. Sete $(58,3 \%)$, dentre 12 pacientes com 70 anos ou mais, foram a óbito.

Dos 12 pacientes operados no mesmo dia da rotura septal, $6(50 \%)$ faleceram; dos 12 operados de 1 a 7 dias pós RSI, 6 (50\%) faleceram; 2 pacientes foram operados entre 7 e 15 dias pós RSI, sem mortalidade; 6 foram operados entre 15 e 30 dias, com $4(66,6 \%)$ óbitos: 6 operados depois de 30 dias sobreviveram; em 4 pacientes, esse intervalo não foi determinado (Tabela 1).

Pudemos associar mortalidade mais elevada ao maior número de artérias lesadas. Dentre 20 pacientes com lesão uniarterial, $5(25 \%)$ faleceram; de $10 \mathrm{com}$ duas artérias lesadas, $4(40 \%)$ faleceram e, nos 12 restantes, com comprometimento triarterial, $7(58,3 \%)$ foram a óbito.

Quatorze (33,3\%) pacientes foram revascularizados no mesmo ato cirúrgico, tendo $8(57,1 \%)$ recebido uma ponte de veia safena, dos quais $2(25 \%)$ faleceram; 5

TABELA 1

INTERVALO ENTRE ROTURA SEPTAL E OPERACAOOMORTALIDADE

\begin{tabular}{lccc}
\hline$\Delta t$ - RSI e Operação & N: Pacientes & Mritalidade & $\%$ \\
\hline MESMO DIA & 12 & 6 & $50 \%$ \\
1 a 7 dias & 12 & 6 & $50 \%$ \\
7 a 15 dias & 2 & - & $0 \%$ \\
15 a 30 dias & 6 & 4 & $66,6 \%$ \\
na de 30 dias & 6 & - & $0 \%$ \\
não determinado & 4 & - & $0 \%$ \\
\hline
\end{tabular}

$(35,7 \%)$ receberam duas pontes de veia safena, com $3(60 \%)$ óbitos; e $1(7,1 \%)$ recebeu três pontes de safena, tendo sobrevivido.

Dos $28(66,6 \%)$ pacientes com RSI de localização anterior, $9(32,1 \%)$ faleceram; dos $14(33,3 \%)$ cuja rotura localizava-se na porção posterior do septo, 7 (50\%) faleceram.

Dos 30 pacientes cujo acesso à CIV foi através de ventriculotomia E, $22(73,3 \%)$ eram portadores de RSI anterior, dos quais $7(31,9 \%)$ faleceram. Em $8(26,6 \%)$, a RSI era posterior, tendo havido $6(75 \%)$ óbitos. Nos outros 12 pacientes, as cavidades ventriculares D e E foram expostas; 6 pacientes desse grupo apresentavam $\mathrm{RSI}$ anterior, dos quais $2(33,3 \%)$ faleceram. Outros 6 foram portadores de RSI posterior, tendo havido 1 $(16,6 \%)$ óbito. (Tabela 2).

Vinte e um pacientes foram operados em boas condiçōes hemodinâmicas. Neste grupo, apenas $2(9,5 \%)$ faleceram. Dos 4 pacientes operados com choque cardiogênico instalado a menos de 12 horas, $1(25 \%)$ faleceu; de 10 pacientes cujos sinais de choque cardiogênico datavam de 12 a 24 horas, $7(70 \%)$ faleceram e de 7 pacientes com choque cardiogênico presente há mais de 24 horas, $6(85,7 \%)$ foram a óbito. (Tabela 3$)$.

Quatro $(9,5 \%)$ pacientes faleceram no intra-operatório; $9(21,4 \%)$ no pós-operatório imediato (até 2: P.O.) e $3(7,1 \%)$ no pós-operatório tardio (hospitalar).

As principais complicaçōes dos 38 pacientes que chegaram à sala de recuperação cardiaca pós-operatória foram:

$\begin{array}{lrr}\text { - síndrome de baixo débito cardiaco } & 28(73,7 \%) \\ \text { — déficit neurológico } & 11(28,9 \%) \\ \text { - insuficiência renal aguda } & 9(26,7 \%) \\ \text { - insuficiência respiratória } & 6(15,8 \%) \\ \text { - isquemia mesentérica } & 3(7,9 \%) \\ \text { - sepsis } & 2(5,2 \%) \\ \text { - mediastinite } & 2 & (5,2 \%) \\ \text { - sangramento necessitando reoperação } & 2 & (5,2 \%) \\ \text { - bloqueio atrioventricular total } & 2(5,2 \%) \\ \text { - isquemia de membros inferiores } & 1(2,6 \%)\end{array}$

\section{COMENTÁRIOS}

O tratamento da CIV pós IAM é essencialmente cirúrgico. A perfuração septal súbita expõe o ventrículo direito, desadaptado e muitas vezes infartado, à pressão da câmara ventricular $\mathrm{E}$. Isso acarreta grande aumento do fluxo sangüíneo pulmonar e conseqüente disfunção do $\mathrm{VD}^{33}$. A pressão diastólica final de VE mais elevada que a de VD faz com que o shunt E-D persista também durante a diástole, contribuindo ainda mais para a falência de VD. Por outro lado, o roubo de fluxo através da CIV leva à diminuição do débito sistêmico efetivo de VE. Como decorrência, há maior recirculação sangüínea 
DALLAN, L. A.; OLIVEIRA, S. A.; RAMIRES, J. A. F.; SABINO NETO, A.; VERGINELLI, G.; JATENE, A. D. - Tratamento cirúrgico da comunicação interventricular pós infarto agudo do miocárdio: conduta atual. Rev. Bras. Cir. Cardiovasc., 4(1): 64-74, 1989.

TABELA 2

TÉCNICA OPERATORIA / LOCALIZAÇÃO DA RSI/MORTALIDADE

\begin{tabular}{lcccc}
\hline Acesso ao Septo IV & Localização CIV & N. de Pacientes & Mortalidade & \% \\
\hline Ventriculotomia E & | anterior & $22(73.3 \%)$ & 7 & $31,9 \%$ \\
Exposterior & $8(26,6 \%)$ & 5 & $75 \%$ \\
& |anterior & $6(50 \%)$ & 2 & $33,3 \%$ \\
\hline
\end{tabular}

TABELA 3

CONDIÇĀO HEMODINAMICA PRÉ-OPERATORIA / MORTALIDADE

\begin{tabular}{|c|c|c|c|c|}
\hline \multicolumn{2}{|c|}{ At Choque Cardogénico } & No de Pacientes & Mortalidade & $\%$ \\
\hline Sem Choque Cardiog & & 21 & 2 & $9,5 \%$ \\
\hline & $12 \mathrm{hs}$ & 4 & 1 & $25 \%$ \\
\hline \multirow[t]{2}{*}{ Choque cardiogênico } & $12-24 \mathrm{hs}$ & 10 & 7 & $70 \%$ \\
\hline & $1>24$ hs & 7 & 6 & $85,7 \%$ \\
\hline Total & & 42 & 16 & $38,1 \%$ \\
\hline
\end{tabular}

pulmonar e conseqüente aumento no retorno sangüíneo ao VE recém-infartado, também predispondo sua falên$\mathrm{cia}^{24}$. Essas evidências traduzem a gravidade dessa complicação do IAM.

COOLEY et alii ${ }^{2}$, em 1957 realizaram a primeira correção cirúrgica da rotura septal pós infarto. Desde então, inúmeros trabalhos se sucederam, demonstrando a necessidade do tratamento cirúrgico da RSI, dada a elevada mortalidade nos primeiros dias de sua instalação ${ }^{25}$, 29. 33 . Menos de $10 \%$ dos pacientes não operados resistem ao primeiro ano pós RSI $I^{1,15}$ e são poucos os relatos de pacientes que sobreviveram vários anos sem correção cirúrgica ${ }^{21,} 36$.

Nossa experiência, acumulada nos últimos anos com o manuseio de pacientes portadores de RSI, nos permitiu estabelecer observaçōes e condutas bem definidas.

A RSI ocorre, preferencialmente, em pacientes brancos, do sexo masculino ${ }^{13}, 18,23$. Em nossa casuistica, a incidência em homens predominou em $61,9 \%$. Não verificamos, entretanto, variação significativa de mortalidade e morbidade em relação ao sexo.

Inúmeros relatos da literatura demonstram que a RSI ocorre (em média) nos primeiros anos da sexta década de vida ${ }^{9}, 13,27,30$. A faixa etária média de nossos pacientes $(65,38$ anos) coincidiu com essas observaçōes. WEINTRAUB et alii ${ }^{40}$ obtiveram $42 \%$ de mortalidade hospitalar dentre 12 pacientes idosos $(M=72$ \pm 5 anos) submetidos a correçāo cirúrgica da RSI, considerando-a aceitável. Nossa casuística, ao contrário, constatou que a mortalidade elevou-se de $30 \%$ nos pa- cientes em faixa etária inferior a 70 anos, para $58,3 \%$ naqueles com 70 anos ou mais, demonstrando a maior gravidade no manuseio de pacientes idosos ${ }^{35}$.

Todos os nossos pacientes foram estudados através de exame cinecoronariográfico. Isto possibilitou a confirmação de suspeita clínica e quantificou sua repercussão hemodinâmica, permitindo definir, com exatidão, a extensão das lesões coronarianas.

O advento da ecocardiografia bidimensional e Doppler pulsátil tem permitido diagnósticos precoces e não invasivos da RSI, possibilitando sua distinção com a regurgitaçāo mitral decorrente da rotura do músculo papilar ${ }^{39}$. O ecocardiograma, associado ao ECG e aos dados obtidos através de cateteres de Swan-Ganz, permite identificar a parede ventricular infartada ${ }^{19}$ e quantificar o shunt E-D. Nos pacientes mais graves, pode-se, portanto, dispensar os exames mais invasivos e abreviar o período pré-operatório ${ }^{5}$. Por outro lado, não está comprovada a melhora na sobrevida hospitalar com a associação de revascularização miocárdica desses pacientes $^{4}, 14,19,22,23,29,40$. Quatorze $(33,3 \%)$ de nossos pacientes receberam de 1 a 3 pontes de veia safena durante a intervenção cirúrgica, com $5(35,7 \%)$ obitos. Esses valores não nos possibilitaram definir, estatisticamente, vantagens na revascularização simultânea do miocárdio.

MALLORY \& WHITE ${ }^{24}$ descreveram a fisiopatologia da RSI através da desintegração do músculo necrótico, na segunda semana pós IAM. As alterações inflamatórias locais e as altas concentraçōes de enzimas proteolíticas, associadas à movimentação paroxística entre o músculo saudável e o infartado, propiciariam sua rotura. Entretanto, em apenas $14,3 \%$ de nossos pacientes, a 
DALLAN, L. A.; OLIVEIRA, S. A.; RAMIRES, J. A. F.; SABINO NETO, A.; VERGINELLI, G.; JATENE, A. D. - Tratamento cirúrgico da comunicaçāo interventricular pós infarto agudo do miocárdio: conduta atual. Rev. Bras. Cir. Cardiovasc., 4(1): 64-74, 1989.

RSI ocorreu na segunda semana pós IAM. Em sua grande maioria $(66,7 \%)$, ela ocorreu na primeira semana do IAM, especialmente no primeiro dia.

A RSI apresenta maior incidência em pacientes com lesão uniarterial ${ }^{14}$, geralmente acometidos por um primeiro infarto transmural ${ }^{15}$, 32 . Explica-se o fato pela redução abrupta do fluxo sangüíneo para artérias septais destituídas de circulação colateral ${ }^{26}$. Nossos dados coincidem com os da literatura, uma vez que $46,7 \%$ dos pacientes apresentavam apenas 1 artéria comprometida. Entretanto, pudemos observar estrita correlação entre maior número de artérias lesadas e mortalidade, o que não tem sido consenso geral ${ }^{29}$. 33 . Em nossa casuística, $25 \%$ dos pacientes com lesão uniarterial faleceram. Esta cifra elevou-se a 40 e $58,3 \%$, respectivamente, em pacientes com comprometimento bi e triarterial.

A incidência de aneurisma de VE concomitante com RSI é variável, segundo diferentes relatos. GRAHAM et alli ${ }^{9}$ a consideraram infreqüente, justificando o fato pelo curto período decorrido entre o IAM e a operação. Entretanto, outros autores descreveram elevada associação entre aneurisma de VE e RSI ${ }^{14}$, 32 . Isto seria conseqüente à grande extensão da lesão miocárdica que acompanha a RSI, predispondo o enfraquecimento da parede ventricular ${ }^{17}$. Em $24(57,1 \%)$ pacientes de nossa série, foi possivel a correção simultânea da RSI e do aneurisma de VE. Não observamos variação estatística de mortalidade associada a esse procedimento.

A magnitude do fluxo sangüíneo através da CIV é traduzida pela relação fluxo pulmonar/fluxo sistêmico $(0$ $\mathrm{P} / \mathrm{S})$. SELZER et alii ${ }^{36}$ obtiveram valores médios de 0 $P / S$ variáveis entre 1,9 e 4,3. Vários outros estudos indicam grande oscilação nesses valores, com médias que atingem até $6,6^{20}$. Existem relatos de correlaçāo linear entre o shunt $\mathrm{E}-\mathrm{D}$ e o tamanho da $\mathrm{CIV}^{12}$. Entretanto, o emprego da relação $0 \mathrm{P} / \mathrm{S}$ como prognóstico de sobrevida na RSI vem sendo questionado. MOORE et alii ${ }^{20}$ e REDFORD et alii ${ }^{33}$ encontraram valores de $\emptyset \mathrm{P} / \mathrm{S}$ semelhantes entre sobreviventes e pacientes que faleceram em decorrência da RSI. Essa relação também não teve variação estatística entre pacientes com infarto de paredes anterior e inferior. SCANLON et alii ${ }^{35}$ observaram menores valores da relação $0 \mathrm{P} / \mathrm{S}$ nos pacientes que sobreviveram à correção da RSI em relação aos demais. FENELEY et alii ${ }^{6}$, paradoxalmente, observaram médias mais elevadas de $0 \mathrm{P} / \mathrm{S}$ nos pacientes sobreviventes. Os autores atribuíram esses resultados à má função ventricular $E$ nos pacientes que faleceram. A relação $0 \mathrm{P} / \mathrm{S}$ cbtida em 17 de nossos pacientes variou de 1,74 a 5,87 ( $M=3,06)$. Pudemos observar correlação significativa entre valores mais elevados de $O$ PS e maior intensidade dos sintomas clínicos. O mesmo não ocorreu, entretanto, com a mortalidade, cuja maior incidência independeu dos valores de fluxo pulmonar e shunt E-D.

A determinação da saturação de oxigênio em amostras sangüineas obtidas pelo cateterismo direito possi- bilitou detectar salto oximétrico entre AD e AP. Os valores médios verificados em $A D$ e AP foram, respectivamente, $61,50 \%$ e $82,33 \%$, bastante semelhantes aos observados na literatura ${ }^{31}$.

O momento ideal para a intervenção cirúrgica foi motivo de controvérsias. Na década passada, GIULIANI et alii ${ }^{8}$ preconizaram aguardar 3 a 6 semanas para a correçāo da RSI. SCHUMAKER ${ }^{37}$ também sugeriu 0 tratamento conservador inicial, considerando a dificuldade de se suturarem tecidos recém-necrosados e o elevado risco de deiscência. Entretanto, observamos que os bons resultados obtidos com a correção cirúrgica, nesse período, contrastaram com a elevada mortalidade pré-operatória. Atualmente, a grande maioria dos autores é favorável à intervenção cirúrgica precoce após a $\mathrm{RSI}^{3}, 4,9,14,20,23,25,28,29,30-32$.

Também compartilhamos dessa opinião. Nossa elevada mortalidade entre os pacientes operados na primeira semana pós RSI ( $50 \%)$, é justificada pela precariedade em seu estado pré-operatório. Dentre 21 pacientes operados com choque cardiogênico instalado, 14 $(66,6 \%)$ faleceram. Esa cifra caiu para apenas $9,5 \%$ entre os demais operados em boas condiçōes hemodinâmicas. Além do mais, observamos que a persistência do estado de choque elevou, progressivamente, a mortalidade: $25 \%$ dos pacientes operados, nas primeiras 12 horas, de choque cardiogênico faleceram; esta cifra subiu para 70 e $85,7 \%$, quando os sinais de choque datavam, respectivamente, $12-24$ horas e mais de 24 horas.

A correção cirúrgica da rotura septal de localização anterior ou apical é tecnicamente mais fácil e propicia melhores resultados do que a posterior ${ }^{28,23,14}$. A elevada taxa de mortalidade na RSI de localização posterior está bem documentada na literatura ${ }^{4}$. ${ }^{30}$. A razão principal é a grande extensão das estruturas anatômicas envolvidas no IAM, especialmente de VD ${ }^{27}$. O músculo papilar posterior da valva mitral e as inserçōes septais da valva tricúspide são, também, potencialmente vulneráveis ${ }^{23}$.

Inúmeras técnicas foram propostas para o fechamento das CIVs de localização anterior e posterior. DAG$\mathrm{GET}^{3}$, em sucessivas publicações, culminou por sugerir o reforço de ambas as faces do septo IV com feltro de Dacron, seguido, ou não, de ampliação da parede livre do VE. GUNDRY et alii ${ }^{11}$ demonstraram, em estudo experimental, vantagens na rotação de segmento da parede do VD sobre o septo roto, fechando-o e preservando a função do VE.

A proteção miocárdica durante a correção da RSI tem sido variável. A maioria dos autores utiliza hipotermia profunda $\left(20^{\circ} \mathrm{C}\right)$ e cardioplegia ${ }^{3,22,27,40}$. Em nosso Serviço, temos dado preferência pela correção da CIV com pinçamento aórtico apenas durante a ventriculotomia, evitando proporcionar isquemias adicionais ao miocárdio já comprometido. A revascularizaçāo miocárdica, quando associada à correção da RSI, tem sido realizada com 
DALLAN, L. A.; OLIVEIRA, S. A.; RAMIRES, J. A. F.; SABINO NETO, A.; VERGINELLI, G.; JATENE, A. D. - Tratamento cirúrgico da comunicaçăo interventricular pós infarto agudo do miocárdio: conduta atual. Rev. Bras. Cir. Cardiovasc., 4(1): 64-74, 1989.

hipotermia de $32^{\circ} \mathrm{C}$ e pinçamento aórtico intermitente por pequenos períodos.

Doze pacientes de nossa série foram operados, recentemente, utilizando-se técnica que permite a exposiçāo das cavidades ventriculares $D$ e $E$ através da ampliaçăo da CIV, ou biventriculotomia. O revestimento de ambas as faces do septo com tecido biológico tem proporcionado seu reforço adequado. Seis pacientes com RSI anterior foram operados por esta técnica, 2 (33,3\%) dos quais faleceram. Este indice foi semelhante ao observado nos demais 22 pacientes com RSI anterior corrigida apenas por ventriculotomia $\mathrm{E}(31,9 \%)$. Entretanto, essa técnica tem proporcionado resultados bastante satisfatórios no tratamento da RSI de localização posterior. Dentre 6 pacientes operados com esse tipo de abordagem cirúrgica, apenas $1(16,6 \%)$ faleceu, taxa significativamente inferior à observada até então, quando 6 (75\%), dentre 8 pacientes com RSi posterior, haviam falecido.

As complicaçōes pós-operatórias mais freqüentes, como o baixo débito cardiaco, déficit neurológico e insuficiência renal aguda, decorreram basicamente da má condição hemodinâmica que antecedeu a correção cirúrgica.

Dois pacientes apresentaram CIV residual e 1, pseudo-aneurisma no local de sutura do VE. Os 3 foram reoperados já em fase crônica, com sucesso.

Os resultados cirúrgicos tardios têm sido excelentes. Vinte e quatro $(92,3 \%)$ dos 26 pacientes que receberam alta hospitalar encontram-se assintomáticos.

A elevada mortalidade dos pacientes em choque cardiogênico prolongado nos tem conduzido a intervençōes cirúrgicas precoces, se possivel assim que estabelecido o diagnóstico. Por outro lado, acreditamos que a estabilização hemodinâmica obtida através de drogas vasodilatadoras e o uso de balão intra-aórtico nẫo deva ser motivo para se postergar a operaçāo. Pacientes com sinais iniciais de baixo débito cardiaco devem ser tratados como se já estivessem em choque cardiogênico.

A correção da CIV pós IAM através da técnica de exposição biventricular e reforço bilateral do septo tem demonstrado menor mortalidade e ausência de recidivas, especialmente na RSI de localização posterior.

RBCCV $44205-78$

DALLAN, L. A.; OLIVEIRA, S. A.; RAMIRES, J. A. F.; SABINO NETO, A.; VERGINELLI, G.; JATENE, A. D. - Present surgical management of post-myocardial infarction ventricular septal rupture. Rev. Bras. Cir. Cardiovasc., 4(1): 64-74, 1989.

ABSTRACT: The authors describe the experience with surgical treatment of 42 patients with ventricular septal rupture (VSR) in post acute myocardial infarction (AMI). They showed increased mortality in patients with cardiogenic shock $(66.6 \%)$ with respect to others $(9.5 \%)$. Sudden descompensations in patients hemodinamically stable were indications to early surgeries, if possible when the diagnosis of ventricular septum rupture (VSR) was made. The compromise of multiple coronary arteries and advanced age were considered as aggravating factors in the prognosis of surgery. The same prognosis did not occur regarding pulmona$\mathrm{ry} /$ systemic flow and shunt E-D. The technique of exposure in both ventricular cavity and reinforcement in the both sides of the ruptured septum with biological tissue offered good results in the correction of posterior VSR. surgery.

DESCRIPTORS: ventricular disruption, surgery; ventricular septal defect, surgery; myocardial infarct,

\section{REFERÊNCIAS BIBLIOGRÁFICAS}

1 BARNARD, P. M. \& KENNEDY, J. H. - Postinfarction ventricular septal defect. Circulation, 32: 76-83, 1965.

2 COOLEY, D. A.; BELMONTE, B. A.; ZEIS. L. B.; SCHNUR, S. - Surgical repair of ruptured interventricular septum following myocardial infarction. Surgery, 41: 930. 937, 1957.

3 DAGGETT, W. M. - Surgical technique for early repair of posterior ventricular septal rupture. J. Thorac. Cardiovasc. Surg., 84: 306-312, 1982.
4 DAGGETT, W. M.; GUYTON, R. A.; MUNDTH, E. D.; BUCKLEY, M. J.; McENANY, M. T.; GOLD, H. K.; LEINBACH, R. O. C.; AUSTEN, W. G. - Surgery for postmyocardial infarction ventricular septal defect. Ann. Surg., 186: 260-271, 1977.

5 FARCOT, J. C.; BOISANTE, L.; RIGAUD, M.; BARDET, J.; BOURDARIAS, J. P. - Two dimensional echocardiographic visualization of ventricular septal rupture after acute anterior myocardial infarction. Am. J. Cardiol., 45: 370-377, 1980.

6 FENELEY, M. P.; CHANG, V. P.; O'ROURKE, M. F. Myocardial rupture after acute myocardial infarction: ten years review. Br. Heart J., 49: 550-556, 1983. 
DALLAN, L. A.; OLIVEIRA, S. A.; RAMIRES, J. A. F.; SABINO NETO, A.; VERgINELLI, G.; JATENE, A. D. - Tratamento cirúrgico da comunicaçāo interventricular pós infarto agudo do miocárdio: conduta atual. Rev. Bras. Cir. Cardiovasc., 4(1): 64-74, 1989.

7 FORX, A. C.; GLASSMAN, E.; ISOM, O. W. - Surgically remediable complications of myocardial infarction. Prog. Cardiovasc. Dis., 21: 461-469, 1979.

8 GIULIANI, E. R.; DANIELSON, G. K.; PLUTH, J. R.; ODYNIEC, N. A.; WALLACE, R. B. - Postinfarction ventricular septal rupture. Circulation, 49: 455-459, 1974.

9 GRAHAM, A. F.; STINSON, E. B.; DAILY, P. O.; HARRISON, D. C. - Ventricular septal defects after myocardial infarction: early operative treatment. JAMA, 225: 708-711, 1978.

10 GARY, R. J.; SETHNA, D.; MATLOFF, J. M. - The role of cardiac surgery in acute myocardial infarction with mechanical complications. Am. Heart J., 10 a. 723-728, 1983.

11 GUNDRY, S. R.; COUCHLIN, T. R.; GOLDBERG, N. H.; HANKINS, J. R.; MACKENZIE, C. F.; FLOWERS, J.; MOORMAN, R.; McLAUGHLIN, J. S. - Experimental repair of ventricular septal defects using autologous right ventricular muscle flaps: preliminary report. Ann. Thorac. Surg., 46: 278-282, 1988.

12 HEIKKILÄ, J.; KARESOJA, M.; LUOMANMÄKI, K. - Ruptured interventricular septum complicating acute myocardial infarction. Chest, 66: 675-681, 1974.

13 HEITMILLER, R.; JACOBS, M. L.; DAGGETT, W. M. Surgical management of postinfarction ventricular septal rupture. Ann. Thorac. Surg., 41: 683-691, 1986.

14 HILL, J. D.; LARY, D.; KERTH, W. J.; GERBODE, F. Acquired ventricular septal defects. J. Thorac. Cardiovasc. Surg., 70: 440-450, 1975.

15 HUTCHINS, G. M. - Rupture of the interventricular septum complicating myocardial infarction: pathological analysis of 10 patients with clinically diagnosed perforations. Am. Heart J., 97: 165-173, 1979.

16 IBEN, A. B.; PUPELlO, D. F.; STINSON, E. B.; SHUMWAY, N. E. - Surgical treatment of postinfarction ventricular septal defects. Ann. Thorac. Surg., 8: 252-259, 1969.

17 JAVID, H.; HUNTER, J. A.; NAJAFI, H.; DYE, W. S.; JULIAN, O. C. - Left ventricular approach for the repair of ventricular septal perforation and infarctetomy. $J$. Thorac. Cardiovasc. Surg., 63: 14-24, 1972.

18 JULIARD, J. M.; DONZEAN-GOUGE, P.; MASQUET, C. PIWNICA, A.; BEAUFILS, P. - Évolution à long terme des ruptures septales opérées à la phase aigūe d'un infarctus du myocarde. Arch. Mal. Coeur, 79: 333-337, 1986.

19 KAPLAN, M. A.; HARRIS, C. N.; KAY, J. H.; PARKER, D. P.; MAGRIDSON, O. - Postinfarction ventricular septal rupture: clinical approach and surgical results. Chest, 69: 734-738, 1976.

20 KITAMURA, S.; MENDEZ, A.; KAY, J. H. - Ventricular septal defect following myocardial infarction: experien- ce with surgical repair through a left ventriculotomy and review of literature. J. Thorac. Cardiovasc. Surg., 61: 186-199, 1971.

21 LANDON, D. G. \& SCHLAPPI, J. C. - Thirteen-year survival with acquired interventricular septal defect after myocardial infarction. Am. Heart J., 64: 33-37, 1962.

22 LOISANCE, D. - Rupture septale recente après infarctus du myocarde: traitement chirurgical. Rev. Port. Cardiol., 4: 23-24, 1985.

23 LOISANCE, D. Y.; CACHERA, J. P.; POULAIN, H.; AUBRY, P.; JUVIN, A. M.; GALEY, J. J. - Ventricular septal defect after acute myocardial infarction: early repair. J. Thorac. Cardiovasc. Surg., 80:61-67, 1980.

24 MALLORY, G. K. \& WHITE, P. D. - The speed of healing of myocardial infarction: a study of the pathologic anatomy in 72 cases. Am. Heart J., 18: 647-671, 1939.

25 MATLOFF, J. M. \& GRAY, R. J. - Cardiac surgery in myocardial infarction. In: PARMLEY, W. W. \& CHATTERJEE, K. (eds.) Cardiology. Philadelphia, JB Lippincott Company, 1987. Vol. 2, cap. 18, p. 12-13

MILLER, S. W.; DINSMORE, R. E.; DAGGETT, W. G. Coronary, ventricular and pulmonary abnormalities associated with rupture of the interventricular septum complicating myocardial infarction. $A m$. J. Roentgenol., 131: 571-577, 1978.

27 MIYAMOTO, A. T.; LEE, M. E.; KASS, R. M.; CHAUX, A.; SETHNA, D.; GRAY, R.; MATLOFF, J. M. - Post myocardial infarction ventricular septal defect. $J$. Thorac. Cardiovasc. Surg., 86: 41-46, 1983.

28 MONTOYA, A.; MCKEEVER, L.; SCANLON, P.; SULLIVAN, H. J.; GUNNAR, W. M.; PIFARRÉ, R. - Early repair of ventricular septal rupture after infarction. Am. J. Cardiol., 45: 345-349, 1980

29 MOORE, C. A.; NYGAARD, T. W.; KAISER, D. L.; COOPER, A. A.; GIBSON, R. S. - Postinfarction ventricular septal rupture: the importance of location of infarction and right ventricular function in determining survival. Circulation, 74: 45-55, 1986.

30 NAIFEH, J. G.; GREHL, T. M.; HURLEY, E. J. - Surgical treatment of postmyocardial infarction ventricular septal defects. J. Thorac. Cardiovasc. Surg., 79: 483-488, 1980.

31 NESRALLA, I. A.; LUCCHESE, F. A.; PRATES, P. R.; KA LIL, R. K.; SANT'ANNA, J. R.; PEREIRA, E.; LARA, R. F.; COSTA, A. R.; DAUDT, N. S. - Rotura do septo ventricular após infarto agudo do miocárdio. Arq. Bras. Cardiol., 43: 377-380, 1984.

32 PIEGAS, L. S.; SANTOS, R. J.; MIRANDA, M. A. T.; BEMBOM, J. C.; TIMERMAN, A.; BARBOSA, M. A. O.; EGITO, E. T.; ABDULMASSIH NETO, C.; SOUZA, L. C. B.; PAULISTA, P. P.; JATENE, A. D. - Ruptura do septo interventricular pós infarto do miocárdio: o dilema 
DALiAN, L. A.; OLIVEIRA, S. A.; RAMIRES, J. A. F.; SABINO NETO, A.; VERGINELLI, G.; JATENE, A. D. - Tratamento cirúrgico da comunicação interventricular pós infarto agudo do miocárdio: conduta atual. Rev. Bras. Cir. Cardiovasc., 4(1): 64-74, 1989.

da indicação cirúrgica. Arq. Bras. Cardiol., 37: 241-246, 1981.

REDFORD, M. J.; JOHNSON, R. A.; DAGGETT, W. M.; FALLON, J. T.; BUCKLEY, M. J.; GOLD, H. K.; LEIN$\mathrm{BACH}, \mathrm{R}$. C. - Ventricular septal rupture: a review of clinical and physiologic features and an analysis of survival. Circulation, 64: 545-553, 1981

34 ROBERTS, W. C.; RONAN Jr., J. A.; HARWEY, W. P - Rupture of the left ventricular free wall (LVFW) or ventricular septum (VS) secondary to acute myocardial infarction (AMI): an occurrence virtually limited to the first transmural AMI in a hypertensive individual. $A m$. J. Cardiol., 35: 166, 1975. (Resumo).

35 SCANLON, P. J.; MONTOYA, A.; JOHNSON, S. A.; McKEEVER, L. S.; SULLIVAN, H. J.; BANKOS, M.; PIFARRÉ, M.; PIFARRÉ, R. - Urgent surgery for ventricular septal rupture complicating acute myocardial infarction. Circulation, 72 (Supl. 2): 185-190, 1985.

36 SELZER, A.; GERBODE, F.; KERTH, W. J. - Clinical, hemodynamic and surgical considerations of rupture of the ventricular septum after myocardial infarction. Am. Heart J., 78: 598-607, 1969.

37 SCHUMAKER, H. B. - Suggestions concerning operative management of postinfarction septal defects. J. Tho rac. Cardiovasc. Surg., 64: 452-459, 1972.

38 SHAH, P. K. \& SWAN, H. J. C. - Complications of acute myocardial infarction. In: PARMLEY, W. W. \& CHATERJEE, K. (eds.) Cardiology, Vol. 2. cap. 13, p. 22-23.

39 SILVA, M. N.; QUININHA, J.; RAMOS, J. S.; FERREIRA, T.; SALOMĀO, S. - Contribuiçāo da ecocardiografia bidimensional e da ecocardiografia Doppler pulsátil para o diagnóstico das roturas septais no enfarte agudo do miocárdio. Rev. Port. Cardiol., 7: 37-41, 1988.

40 WEINTRAUB, R. M.; THURER, R. L.; WEI, J.; AROESTY, J.M. - Repair of postinfarction ventricular septal defect in the elderly: early and long-term results. J. Thorac. Cardiovasc. Surg., 85: 191-196, 1983.

\section{Discussão}

\section{DR. LEOPOLDO S. PIEGAS \\ São Paulo, SP}

Em nome do Instituto Dante Pazzanese de Cardiologia e em meu próprio, agradeço o convite para comentar este trabalho apresentado pelo Dr. Dallan, que resume os conhecimentos atuais sobre esta complicação do infarto do miocárdio. Foi relatado de forma brilhante e quero me congratular com o autor e seu grupo. Como não sou cirurgião, não vou me atrever a fazer comentários cirúrgicos. Apenas ressaltarei pontos que, para mim, como clíniro. parecem importantes e que vêm con- firmar muito do que foi aqui apresentado. Na experiência do IDPC, não observamos pacientes com condições circulatórias normais; todos apresentavam grave falência ventricular graus funcionais II e III. Observamos, ainda, presença de lesāo uniarterial em $60 \%$ desses pacientes, o que sugere que năo são pacientes terminais ou em fase avançada de evolução da doença coronária. $\mathrm{Na}$ maioria deles, este é o primeiro evento coronário. Tivemos apenas 1 paciente que apresentava infarto prévio, fato que a literatura tem ressaltado muito. Outra observaçāo que gostaria de destacar é a maior mortalidade no infarto de localizaçāo inferior. Estudo recente realizado na Inglaterra, com revisão de 60 trabalhos, atribui ao ventrículo direito uma parcela muito grande na mortalidade pós-operatória desses pacientes. Em nosso Serviço, foram operados 35 pacientes, de um grupo de 54 . Procuraremos demonstrar as características de nosso grupo e o material que foi apresentado pelo Dr. Dallan. Foi observada diferença estatisticamente significativa em relação ao sexo. Nāo houve diferença em relação à localização do infarto, quer seja de parede anterior ou inferior, quer em presença de lesão uniarterial ou multiarterial. Os aneurismas associados apareceram em $54 \%$ no nosso material e em $57 \%$ no material do Dr. Dallan. No que se refere à mortalidade, nossos dados confirmam o que o Dr. Dallan já disse. A literatura tem ressaltado que pacientes com infarto inferior e rotura do septo têm maior incidência de óbito em relação a pacientes cuja rotura tem posição anterior. Em relação à cirurgia precoce, tivemos $58 \%$ dos operados com menos de 30 dias, contra $50 \%$ dos aqui apresentados. A mortalidade global do nosso material foi de $34 \%$. A época da cirurgia talvez seja o ponto mais importante a ser discutido. No ano de 1981, já tivemos a oportunidade de discutir esse assunto, denominado "o dilema da indicação cirúrgica", neste mesmo Congresso da SBCCV. Isto porque sabemos que, se operarmos precocemente haverá maior mortalidade. Entretanto, a gravidade desta lesão não nos permite esperar; portanto, somos de opi. nião que esses pacientes têm que ser operados imediatamente, pois raramente conseguem sobreviver a 30 dias de evolução clínica. Em relação à evolução tardia, dentre 19 pacientes com tratamento clínico, tivemos a mortalidade de quase $90 \%$ dentro de 30 dias, e apenas 1 pacientes sobreviveu, próximo a 1 ano. Ressalvo que esse paciente já chegou ao nosso Serviço com esse período de evolução. Apesar da alta mortalidade imediata, a sobrevida do paciente cirúrgiso é boa, assim como a expectativa de vida. Temos pacientes sobreviventes com 12 anos de evolução. Gostaria de encerrar destacando a variação na incidência de rotura de septo pós infarto nos diferentes qüinqüênios. 0 primeiro caso diagnosticado em nosso Serviço foi em 1968, e, nesses qüinqüênios, tivemos, respectivamente, $3,12,21,14$ e 4 pacientes com rotura septal. A partir de 1982, houve uma redução nesses pacientes e, no último qüinqüênio, essa incidência está em torno de $3,2 \%$. Acreditamos que essa complicaçāo esteja, realmente, se reduzindo, visto que há uma coincidência com a melhora do tratamento clíni- 
DALLAN, L. A.; OlIVEIRA, S. A.; RAMIRES, J. A. F.; SABINO NETO, A.; VERGINELLI, G.; JATENE, A. D. - Tratamento cirúrgico da comunicaçăo interventricular pós infarto agudo do miocárdio: conduta atual. Rev. Bras. Cir. Cardiovasc., 4(1): 64-74, 1989.

œ, especialmente por diferentes técnicas farmacológicas, que, provavelmente, protegem mais o doente. Mais uma vez, congratulo-me com o Dr. Dallan e seu grupo.

\section{DR. LUIZ ANTONIO RIVETTI}

São Paulo, SP

Cumprimento o Dr. Dallan pela excelente apresentação e gostaria de parabenizar os autores pela magnifica estatística, provavelmente uma das maiores da literatura mundial para um período tão curto de 5 anos. $O$ trabalho merece elogios, pois é muito claro, bem documentado, bem discutido e analisado, tanto sob o ponto de vista técnico, como estatístico e apresenta uma mortalidade que considero baixa, diante da gravidade de uma complicação que é considerada letal. Apesar de cirurgiăo, gostaria de iniciar meus comentários analisando a tentativa de estabilizaçăo clínica desses pacientes com drogas diminuidoras da pós-carga. Utilizamos a bioimpedância para controlar pacientes nessas condiçōes e temos observado, em concordância com os autores, que, principalmente em idosos e diabéticos, o prolongamento dessa terapêutica vasodilatadora tem levado a descompensaçōes abruptas com encharcamento pulmonar, apesar de restrição hídrica, provavelmente por alteração da permeabilidade vascular pulmonar, além do hiperfluxo específico, comprometendo, desta maneira, a indicação ou, mesmo, o resultado cirúrgico. 0 momento da indicação cirúrgica é outro ponto de discussão, mas já está determinado que devemos atuar no ápice do sucesso do tratamento clínico, que, em geral, ocorre na primeira semana. Outro ponto é uma crítica aos autores que, provavelmente por modéstia, não referiram esta técnica de abordagem biventricular como original, fazendo-me gastar muito tempo à procura do autor, sem sucesso. Acredito ter sido esta técnica fundamental para o sucesso do resultado alcançado. Toda técnica tem que propiciar uma correção adequada e, também, evitar recidivas. A proteção bilateral do septo propicia melhor apoio do enxerto sobre o tecido comprometido e friável. O envolvimento do septo com enxertos protege o mesmo contra a infiltração de sangue e, conseqüentemente, deve reduzir as recidivas, fato que foi comprovado, pois observou-se somente $5 \%$ de recidivas contra $12 \%$ a $40 \%$ da literatura. Devemos ressaltar, ainda, o sucesso do tratamento da RSI posterior com taxa de mortalidade de somente $16 \%$, contra estatísticas da literatura que variam de $30 \%$ a $40 \%$. Finalmente, gostaria de fazer algumas perguntas ao Dr. Dallan: 1) achei altos os índices de comprometimento neurológico $(28 \%)$ no pós-operatório. O senhor responsabilizaria somente o baixo débito, ou poderiamos pensar que a gênese da rotura septal teria contribuído para a morbidade?. 2) o balão intra-aórtico, sob o ponto de vista dos autores, é imprescindivel, e qual a correlaçăo do seu uso com a mortalidade? 3) a utilizaçăo de enxerto de pericárdio bovino em um ventrículo esquerdo bastante comprometido com uma CIV grande năo poderia propiciar um baixo débito por disci- nesia septal? 4) a sua casuística mostrou uma incidência de $57 \%$ de combinação de aneurisma de VE com RSI; qual a extensão desses aneurismas e se a presença do mesmo não comprometeu a técnica citada? A RSI é considerada rara; mais raro tem sido levar os pacientes em boas condiçōes para a sala de operações e, talvez, mais raro ainda tem sido levá-los em boas condiçōes para os seus aposentos, no pós-operatório. Quando uma doença é catastrófica, como esta, surgem inúmeras técnicas e discussões sobre sua abordagem terapêutica e, nestes casos, a Medicina lança mão de vacinas. Nós, cirurgiōes cardiovasculares, já podemos contar com a "vacina", no tratamento da RSI pós IAM. Essa "vacina" chama-se trombolítico, que, sem dúvida, diminuirá, consideravelmente, a incidência dessa complicação.

\section{DR. FERNANDO SAMPAIO São Paulo, SP}

Gostaria de parabenizar o Dr. Dallan e demais autores, por trazerem um assunto tão importante como tema. Com o objetivo de definir o melhor momento para indicação cirúrgica dessa lesão, nós revimos, em nosso grupo, seu manuseio cirúrgico. No período de 1974 a 1989 , 16 pacientes foram operados, com rotura do septo interventricular, no Instituto de Doenças Cardiopulmonares E. J. Zerbini, da Beneficência Portuguesa de São Paulo. Os pacientes foram subdivididos em 3 grupos, quanto ao momento da indicaçāo cirúrgica. 01 : grupo era composto por $5(33,2 \%)$ pacientes operados até o 3 : dia após a rotura do septo. O 2 . grupo, formado por 8 (50\%) pacientes operados entre o 4: e o $10^{\circ}$ dia após a rotura do septo. $\mathrm{O} 3$ : grupo compreendia $3(16,8 \%)$ pacientes, com indicação cirúrgica após o 1: mês de rotura do septo. Dos 5 pacientes do 1 : grupo operados, apenas 1 desenvolveu choque cardiogênico e foi a óbito (20\%). No 2 . grupo, durante a espera, 7 dos 8 pacientes desenvolveram choque cardiogênico, dos quais $6(75 \%)$ foram a óbito. A mortalidade total foi de 7 pacientes, dentre os 8 pacientes que estavam em choque cardiogênico, com indicação cirúrgica. Corn base nestes dados, concluímos que a principal determinante do risco cirúrgico, na correção da rotura do septo interventricular, é a presença de choque cardiogênico. A instalação do choque cardiogênico é, às vezes, rápida e ocorre, com freqüência, na 1: semana após o diagnóstico. Na nossa opinião, a indicação cirúrgica deve ser precoce, antes das complicaçōes, ressaltando a presença do choque. Muito obrigado. Quero agradecer a Comissão Organizadora e, mais uma vez. cumprimentar o Dr. Dallan e seus colaboradores.

\section{DR. DALLAN}

(Encerrando)

Inicialmente, gostaria de agradecer os comentários elogiosos dos professores Leopoldo Piegas, Luiz Rivetti 
DALLAN, L. A.; OLIVEIRA, S. A.; RAMIRES, J. A. F.; SABINO NETO, A.; VERGINELLI, G.; JATENE, A. D. - Tratamento cirúrgico da comunicaçāo interventricular pós infarto agudo do miocárdio: conduta atual. Rev. Bras. Cir. Cardiovasc., 4(1): 64-74, 1989.

e Fernando Sampaio. Dr. Leopoldo Piegas, foi com satisfação que verifiquei os excelentes resultados do Instituto Dante Pazzanese de Cardiologia, no tratamento da CIV pós IAM, o que, mais uma vez, demonstra a ótima qualidade do Serviço a que o senhor pertence. Pude observar grande superposição entre seus dados estatísticos e os nossos, especialmente na correlação entre classe funcional pré-operatória dos pacientes e a mortalidade. Temos, igualmente, evitado postergar a correção cirúrgica, procurando indicá-la o mais precocemente possível. Sua observação de que a mortalidade é maior na CIV de localização mais pcsterior, ou seja, decorrente do infarto de parede inferior, vem de encontro aos achados de literatura e, também, com os nossos resultados iniciais. Recentemente, entretanto, empregamos, nos últimos 6 pacientes com rotura do septo interventricular de localização posterior, a técnica de fechamento da CIV, através da exposição biventricular. Esse artifício possibilitou a redução significativa da mortalidade, que caiu de 75 para $16,6 \%$, tornando a técnica bastante promissora. Dr. Rivetti, considero muito oportuna sua sugestão visando ao controle desses pacientes através da bioimpedância. Realmente, temos encontrado dificuldades no acompanhamento dos mesmos apenas através de critérios clínicos. Corroborando esta afirmação está o fato de doentes estáveis, já na segunda, ou terceira semana de rotura septal, apresentarem descompensação súbita e não detectada a tempo. Isto os transferiu de uma condição clínica favorável, com baixa mortalidade cirúrgica, para - grupo de choque, cuja mortalidade é muito elevada, como já apresentado. Realmente, 11 (28\%) pacientes de nossa casuística apresentaram agitação neuropsicomotora, ou coma superficial no pós-operatório imediato. Isto foi atribuído à importante condição de baixo débito desses pacientes observada no pré-operatório, associada aos efeitos da circulação extracorpórea. Entretanto, em 10 deles, esse episódio foi transitório e apenas 1 paciente persistiu com seqüela neurológica. $O$ balão intra-aórtico (BIA) foi passado em todos os pacientes em choque cardiogênico e nos pacientes que apresentaram dificuldade para a saída de circulaçăo extracorpórea.
Nossa rotina atual, nos pacientes com CIV pós IAM inclui a passagem do cateter de Swan-Ganz e a introduçāo do $\mathrm{BIA}$, se possível ainda no pré-operatório. $\mathrm{O}$ advento de cateteres de menor diâmetro e introduzidos por punção da artéria femoral tornou o método mais rápido $e$ sem a morbidade anteriormente observada. Acredito que este seja o tipo de complicação do IAM em que a contrapulsaçāo com o BIA encontre uma de suas maiores indicaçōes. Dr. Rivetti, temos utilizado, de rotina, retalhos de pericárdio bovino, visando ao reforço do septo interventricular roto. Em geral, empregamos uma placa em cada lado do septo, seja através da biventriculotomia, ou pela ampliação da CIV. Não vejo a possibilidade dessas placas propiciarem discinésia septal, uma vez que sua superfície livre é pequena e o enxerto, em grande parte, acaba incorporado à ventriculografia. Complementando sua questão, podemos dizer que não observamos maior mortalidade nos pacientes que apresentavam aneurisma de VE associado. A técnica empregada nesses pacientes nāo variou, em relação à proposta para a correção da CIV dos demais pacientes. Pelo contrário, a presença de dilatação da cavidade ventricular esquerda facilitou, em muitos casos, o acesso às duas faces do septo interventricular. Nesses pacientes, a operação foi posteriormente complementada pela aneurismectomia do VE. Respondendo ao Dr. Fernando Sampaio, posso afirmar que também indicamos precocemente a correção cirúrgica da rotura do septo interventricular pós IAM. Ela deve ocorrer, se possivel, assim que estabelecido o diagnóstico e tomadas as primeiras medidas, visando melhorar as condições hemodinâmicas do paciente. Pudemos observar que nossa mortalidade elevou-se de $9,5 \%$ nos pacientes ainda estáveis hemodinamicamente, para $66,6 \%$ naqueles operados já com choque cardiogênico instalado. E mais, esta cifra subiu para $70 \%$ e $85,7 \%$, respectivamente, quando os sinais de choque já perduravam por 12 a 24 horas e por mais de 24 horas. Estes números falam por si. Finalizando, agradeço à Comissão Organizadora do 16 : Congresso Nacional de Cirurgia Cardiaca por esta oportunidade. 